\title{
Supply chain coordination for a deteriorating product under stock-dependent consumption rate and unreliable production process
}

\author{
B. C. Giri ${ }^{\mathrm{a}}$ and A. Chakraborty ${ }^{\mathrm{a}}$
}

${ }^{a}$ Department of Mathematics, Jadavpur University, Kolkata 700 032, India

\begin{tabular}{l} 
A R T I C L E I N F O \\
\hline Article history: \\
Received 1 July 2010 \\
Received in revised form \\
2 October 2010 \\
Accepted 4 October 2010 \\
Available online 4 October 2010 \\
\hline Keywords: \\
Supply chain management \\
Machine shift \\
Stock dependent demand \\
deterioration \\
Single-vendor single-buyer \\
Inventory management
\end{tabular}

A B S T R A C T

This article develops a supply chain coordination model with a single-vendor and a singlebuyer. The vendor manufactures the product in lots and delivers to the buyer in equal shipments. The vendor's production process is not perfectly reliable. During a production run, the process may shift from an in-control state to an out-of-control state at any random time and produces some defective items. The buyer whose demand is assumed to be a linear function of the on-hand inventory performs a screening process immediately after each replenishment. Moreover, the buyer's inventory is deteriorated at a constant rate over time. The vendor-buyer coordination policy is determined by minimizing the average cost of the supply chain. It is observed from the numerical study that channel coordination earns significant cost savings over the non-coordinated policy.

\section{Introduction}

The strategic coordination between vendor (supplier) and buyer (retailer) has become one of the key issues in today's supply chain management. It has been recognized and verified in practice that supply chain performance cannot produce satisfactory result without smooth cooperation and collaboration among its members. When a vendor and a buyer are in a mutually agreed contract for a fixed time period, the problem of the buyer is how much quantity to purchase in each order. On the other hand, the vendor has to decide the economic production lot size and the optimal number of shipments to deliver the quantities to the buyer. One of the first works dealing with integrated vendorbuyer model is due to Goyal (1976) who developed a simple supply chain model of single vendor and single customer and their co-ordination. Banerjee (1986) developed a joint economic lot size model where the vendor produces the buyer's shipment size as a separate batch on a lot-for-lot basis assuming that the vendor's production rate is finite. Goyal (1988) relaxed Banerjee's lot-for-lot assumption and proposed a more general economic lot size model to obtain a lower or equal joint total cost. Lu (1995) derived an optimal solution for the single-vendor single-buyer integrated model with equal shipments to the buyer. Goyal (1995) showed that different shipment size policy could give a better solution. His proposed policy involves successive shipments within a production batch increased by a constant factor which is equal to the ratio of production rate over the demand rate. Hill

* Corresponding author. Tel./fax: +91 3324146717

E-mail addresses: bibhas_pnu@yahoo.com (B. C. Giri), ayan.math@gmail.com (A. Chakraborty)

(c) 2011 Growing Science Ltd. All rights reserved.

doi: 10.5267/j.ijiec.2010.07.001 
(1999) derived a structure of the globally optimal batching and shipping policy for the single-vendor single-buyer integrated production inventory problem. Using an interval search approach, Hogue and Goyal (2000) developed a solution procedure for optimal production quantity in a single-vendor single-buyer production-inventory system with unequal and equal sized shipments from the vendor to the buyer under capacity constraint of the transport equipment. Ben-Daya and Hariga (2004) showed that coordination is effective from vendor's as well as buyer's perspectives for stochastic demand and variable lead time. Huang (2004) developed a model to determine an optimal integrated vendor-buyer policy in just-in-time (JIT) environment with unreliability condition. Wee and Chung (2006) proposed a two-echelon distribution-free integrated production-inventory model with imperfect process. They integrated the marketing and manufacturing channels assuming JIT deliveries. Li and Liu (2006) used quantity discount policy to achieve coordination in a two-echelon supply chain where the demand met by the retailer is probabilistic. Kim et al. (2006) determined production allocation and ordering policy in a supply chain consisting of multiple plants and single retailer. Qin et al. (2007) considered volume discounts and franchise fees as coordination mechanism in a system consisting of a supplier and a buyer with price-sensitive demand. Zhou et al. (2008) addressed a twoechelon supply chain coordination model with one manufacturer and one retailer where the demand for the product at the retailer is dependent on the on-hand inventory. They developed the model when the manufacture follows a lot-for-lot policy. Sajadieh et al. (2009) contributed by developing an integrated vendor-buyer model in which the vendor delivers the production batch to buyer in $n$ equal-

sized shipments and the lead time between the vendor and buyer is stochastic. Yang (2010) provided the present value analysis for a similar type of vendor- buyer problem where the lead time is variable and the lead time crashing cost is a polynomial function of the length of lead time.

However, the above mentioned works does not consider the effect of deterioration in retailer's inventory. Deterioration of goods like volatile liquids, fresh vegetables and fruits, radioactive substances, drugs, blood, etc. in the form of direct spoilage or damage, gradual physical decay in course of time, or obsolescence is a natural phenomenon and it has significant impact on the retailer's inventory policy. Ghare and Shrader (1963) first incorporated the possibility of deterioration in inventory modeling. They extended the classical EOQ model with exponentially distributed deterioration to consider a constant deterioration rate over time. A variable rate of deterioration was also considered by Covert and Philip (1973) with Weibull distribution deterioration, Tadikamalla (1978) with Gamma distribution deterioration, Moon and Lee (2000) with Normal distribution deterioration, among others. There is a vast literature on deteriorating or perishable inventory. The readers can be referred to the review articles contributed by Nahmias (1982) and Goyal and Giri (2001) for details.

In this paper, we consider a two-echelon supply chain consisting of a single-vendor and a singlebuyer. The vendor delivers the production lot to the buyer in $n$ shipments. However, the vendor's production process is not perfect. During a production run, it may shift from in-control state to out-of control state at any random time when it produces some defective items. The demand rate at the buyer is assumed as a linear function of the on-hand inventory. The buyer performs a screening process immediately after each replenishment. The objective of the study is to determine the optimal number of shipments from the vendor to the buyer and the optimal order size of the retailer in each replenishment so that the total cost of the supply chain is minimized. The paper is organized as follows. In the next section, assumptions and notations are given. In Section 3, mathematical models are developed from buyer's and vendor's view points and then the supply chain model is constructed. For a numerical example, the proposed supply chain model is demonstrated and the sensitivity analysis is carried out in Section 4. Finally, the paper is concluded in Section 5.

\section{Notations and Assumptions}

The following notations are used throughout the paper. 
I : buyer's stock on display

$d$ : demand rate at the buyer; $d=a+b I$, where $a(\geq 0)$ and $b(>0)$ are real constants

$p$ : vendor's production rate; $p>d$

$n$ : number of shipments from vendor to buyer in each cycle where $n$ is a positive integer

$\tau$ : time interval between successive replenishment at the buyer

$T$ : cycle time; $T=n \tau$

$Q$ : buyer's order quantity for each replenishment

$s_{v}:$ vendor's set up cost per set up

$s_{b}$ : buyer's ordering cost per order

$h_{v}$ : vendor's inventory holding cost per unit of inventory hold

$h_{b}$ : buyer's inventory holding cost per unit of inventory hold

$c$ : cost of unit item

$c_{f}:$ transportation cost per shipment

$c_{s}$ : unit screening cost

$c_{w}:$ warranty cost for each defective item

$x$ : screening rate

$\theta$ : deterioration rate, $0 \leq \theta<1$

$\alpha$ : defective item production rate, $0 \leq \alpha<1$

$t$ : time to process shift

$t_{m}:$ production run time of the vendor

The assumptions made in this paper are as follows:

(i) The supply chain consists of a single-vendor and single-buyer for a single product.

(ii) Buyer's demand rate is a linear function of the on-hand inventory.

(iii) Vendor's production rate is finite and uniform and is greater than the buyer's demand rate.

(iv) During a production run, vendor's production process may shift from an in-control state to an outof-control state at any time due to the occurrence of an assignable cause.

(v) A fraction $(\alpha)$ of items produced after process shift is non-conforming or defective. For each item, a warranty cost $\left(c_{w}\right)$ is incurred to the vendor.

(vi) Vendor's each production batch is transported to buyer in $n$ shipments. 
(vii) Buyer performs a screening process immediately after each replenishment. The screening rate is so high that the number of perfect items is enough to satisfy the demand during screening time.

(viii) Buyer's inventory deteriorates at a constant rate $\theta \quad(0 \leq \theta<1)$. Deteriorated units are neither repaired nor replaced.

(ix) Shortages are not allowed in buyer's inventory.

\section{Proposed Model}

Suppose the time to process shift $t$ at the vendor follows a probability distribution with probability density function $f(t)$. If $E(N)$ denotes the expected number of defective items produced during a production run then we have

$$
E(n)=\alpha \int_{0}^{n Q / p}(n Q-p t) f(t) d t .
$$

$$
\text { Inventory Level Inventory Level }
$$

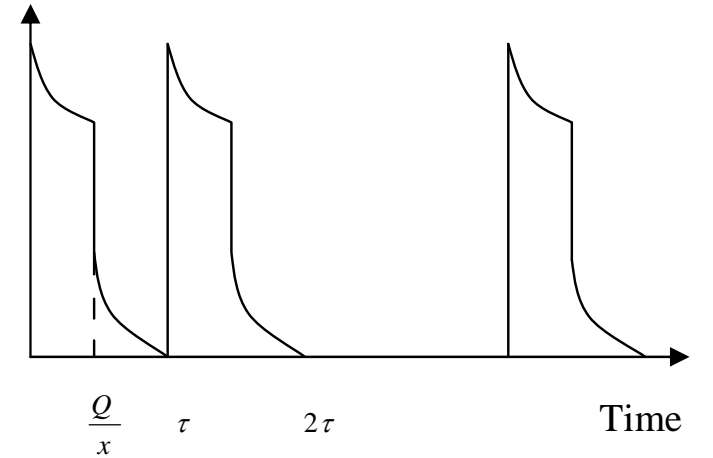

Fig. 1. (a) Buyer's Inventory

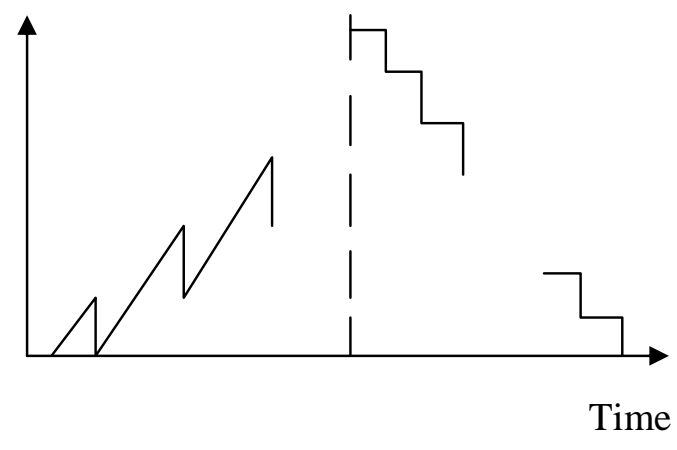

Fig. 1. (b) Vendor's Inventory

\subsection{Buyer's point of view}

The variation of the inventory level at the buyer is depicted in Fig. 1(a). If $I_{i}(t) \quad(i=1,2)$ denotes the inventory level at any time during the time interval $[0, \tau]$, then the instantaneous states of the inventory level are governed by following differential equations:

$\frac{d I_{1}(t)}{d t}+\theta I_{1}(t)=-a-b I_{1}(t), \quad 0 \leq t \leq t_{1}$

with $I_{1}(0)=Q$;

$\frac{d I_{2}(t)}{d t}+\theta I_{2}(t)=-a-b I_{2}(t), t_{1} \leq t \leq \tau$

with $I_{2}(\tau)=0$.

Solving Eq. (2) and Eq. (3) yields, 


$$
\begin{aligned}
& I_{1}(t)=Q \exp (-(\theta+b) t)+\frac{a}{b+\theta}[\exp (-(b+\theta) t)-1], \quad 0 \leq t \leq t_{1} \\
& I_{2}(t)=\frac{a}{b+\theta}[\exp ((b+\theta)(\tau-t))-1], \quad t_{1} \leq t \leq \tau
\end{aligned}
$$

respectively. Since the screening process is completed at a time $t_{1}$, the defective items are expected to be removed from the inventory at that moment. We, therefore, can write $I_{2}\left(t_{1}\right)=I_{1}\left(t_{1}\right)-E(N)$ which gives on simplification

$\tau=\frac{Q}{x}+\frac{1}{b+\theta} \log \left(1+\frac{(b+\theta) R(n, Q)}{a}\right)$,

where $\quad R(n, Q)=Q \exp \left(-(\theta+b) t_{1}\right)+\frac{a}{b+\theta}\left[\exp \left(-(b+\theta) t_{1}\right)-1\right]-E(N)$.

Buyer's holding cost is given by

$$
\begin{aligned}
& h_{b}\left[\int_{0}^{t_{1}} I_{1}(t) d t+\int_{t_{1}}^{\tau} I_{2}(t) d t\right]= \\
& \frac{h_{b}}{b+\theta}\left[\left(Q+\frac{a}{b+\theta}\right)\{1-\exp (-(b+\theta) Q / x)\}+\left(R(n, Q)+\frac{a}{b+\theta}\right)\{1-\exp ((b+\theta)(Q / x-\tau))\}-a \tau\right],
\end{aligned}
$$

and the expected deterioration cost is given by

$$
\begin{aligned}
c\left[Q-E(n)-\int_{0}^{\tau}\left(a+b I_{i}(t)\right) d t\right]= & c\left[Q-E(N)-a \tau-\frac{b}{b+\theta}\left\{\left(Q+\frac{a}{\theta+b}\right)\left(1-\exp \left(-(b+\theta) \frac{Q}{x}\right)\right)\right.\right. \\
& \left.\left.+\left(R(n, Q)+\frac{a}{b+\theta}\right)(1-\exp ((\theta+b)(Q / x-\tau)))-a \tau\right\}\right] .
\end{aligned}
$$

Therefore, the buyer's average total cost which consists of ordering cost for $n$ orders, screening cost, transportation cost, holding cost and deterioration cost is given by

$$
\begin{aligned}
\operatorname{ATC}_{b}(n, Q)= & \frac{s_{b}}{n \tau}+\frac{c_{s} Q+c_{f}}{\tau}+\frac{h_{b}-b c}{(b+\theta) \tau}\left[\left(Q+\frac{a}{b+\theta}\right)\{1-\exp (-(b+\theta) Q / x)\}\right] \\
& +\frac{h_{b}+c}{(b+\theta) \tau}\left[\left(R(n, Q)+\frac{a}{b+\theta}\right)\{1-\exp ((b+\theta)(Q / x-\tau))\}-a \tau\right]+\frac{c}{\tau}[Q-E(N)-a \tau]
\end{aligned}
$$

\subsection{Vendor's point of view}

The variation of the inventory level at the vendor is shown in Fig. 1(b). According to Huang, (2004), the average holding cost of the vendor can be calculated as follows,

$$
\frac{h_{v}}{n \tau}\left[\left\{n Q(Q / p+(n-1) \tau)-\frac{n Q(n Q / p)}{2}\right\}-T\{Q+2 Q+\ldots .+(n-1) Q\}\right]=\frac{h_{v}}{2}\left[(2-n) Q^{2} /(p \tau)+(n-1) Q\right]
$$

The expected warranty cost per unit time is $c_{w} E(N) /(n \tau)$. Therefore, the vendor's average total cost is given by 
$A T C_{v}(n, Q)=\frac{S_{v}}{n \tau}+\frac{h_{v}}{2}\left[(2-n) Q^{2} /(p \tau)+(n-1) Q\right]+\frac{c_{w} E(N)}{n \tau}$.

\subsection{Supply chain model}

From Eq. (9) and Eq. (10), the integrated average total cost for the supply chain is given by

$$
\operatorname{ATC}(n, Q)=\operatorname{ATC}_{b}(n, Q)+A T C_{v}(n, Q) .
$$

Our objective is to determine the optimal number of shipments $n^{*}$ and the optimal order quantity $Q^{*}$ for each shipment so that the average total cost $\operatorname{ATC}(n, Q)$ is minimized.

For exponential process shift distribution $f(t)=\lambda \exp (-\lambda t), \quad 0 \leq t \leq t_{m}$ the necessary condition for optimum of $\operatorname{ATC}(n, Q)$, for given $n$, gives

$$
\begin{aligned}
& \frac{h_{b}-b c}{(b+\theta) \tau}\left[\left\{1-\exp \left(-\left(\frac{b+\theta}{x}\right) Q\right)\right\}+\frac{b+\theta}{x}\left(Q+\frac{a}{b+\theta}\right) \exp \left(-\frac{(b+\theta) Q}{x}\right)\right]+\frac{c_{s}}{\tau}+\frac{h_{b}+c}{(b+\theta) \tau} \\
& \times\left[\frac{d R}{d Q}\left\{1-\exp \left((\theta+b)\left(\frac{Q}{x}-\tau\right)\right)\right\}-\left(R+\frac{a}{\theta+b}\right)(\theta+b) \exp \left((\theta+b)\left(\frac{Q}{x}-\tau\right)\right)-a \frac{d \tau}{d Q}\right]+\frac{c}{\tau}[(1-n \alpha) \\
& \left.-\alpha n \exp \left(-\frac{n \lambda Q}{p}\right)-a \frac{d \tau}{d Q}\right]+\left[\frac{s_{b}}{n}+c_{s} Q+c_{f}+c\left\{(1-n \alpha) Q+\frac{\alpha p}{\lambda}\left\{\exp \left(-\frac{n \lambda Q}{p}\right)-1\right\}-a \lambda\right\}\right. \\
& +\frac{h_{b}+c}{b+\theta}\left\{\left(R+\frac{a}{\theta+b}\right)\left(1-\exp \left((\theta+b)\left(\frac{Q}{x}-\tau\right)\right)\right)-a \tau\right\}+\frac{h_{b}-b c}{b+\theta}\left\{\left(Q+\frac{a}{b+\theta}\right)\left\{1-\exp \left(-\frac{b+\theta}{x}\right) Q\right\}\right] \\
& \times\left(-\frac{1}{\tau^{2}}\right) \frac{d \tau}{d Q}+\frac{1}{\tau}\left[\frac{c_{w}}{n} \frac{d E(N)}{d Q}+\frac{2 Q}{p}\left(1-\frac{n}{2}\right) h_{v}\right]+\frac{n-1}{2} h_{v}+\left[\frac{s_{v}}{n}+\frac{c_{w} E(N)}{n}+\frac{Q^{2}}{p}\left(1-\frac{n}{2}\right) h_{v}\right] \\
& \times\left(-\frac{1}{\tau^{2}}\right) \frac{d \tau}{d Q}=0, \\
& \text { where } \frac{d E(N)}{d Q}=\alpha n[1-\exp (-\lambda n Q / p)] \\
& \frac{d \tau}{d Q}=\frac{1}{x}+\frac{1}{a+(b+\theta) R} \frac{d R}{d Q} \\
& \frac{d R}{d Q}=\left[1-\frac{(b+\theta) Q}{x}-\frac{a}{x}\right] \exp (-(b+\theta) Q / x)-\frac{d E(N)}{d Q} .
\end{aligned}
$$

For a known value of $n$, Eq. (12) can be solved numerically by any one dimensional search method.

\section{Numerical Examples}

To demonstrate the proposed model numerically, we consider the values of the parameters involved in the model as follows:

$a=500, b=0.02, p=800, s_{v}=150, s_{b}=20, h_{v}=0.2, h_{b}=0.4, \theta=0.02, c=10, c_{f}=25, c_{s}=0.5, \alpha=0.05$,

$c_{w}=5, x=1000$ in appropriate units. We obtain the optimal decisions for three different shift distributions as given in the following Examples 1-3. 
Example 1. Exponential process shift distribution with $\lambda=100$

The graph of the cost function $\operatorname{ATC}(n, Q)$ for any given $n$ and a wide range of values of $Q$ is found to be convex curve. One instance is shown in Fig. 2.

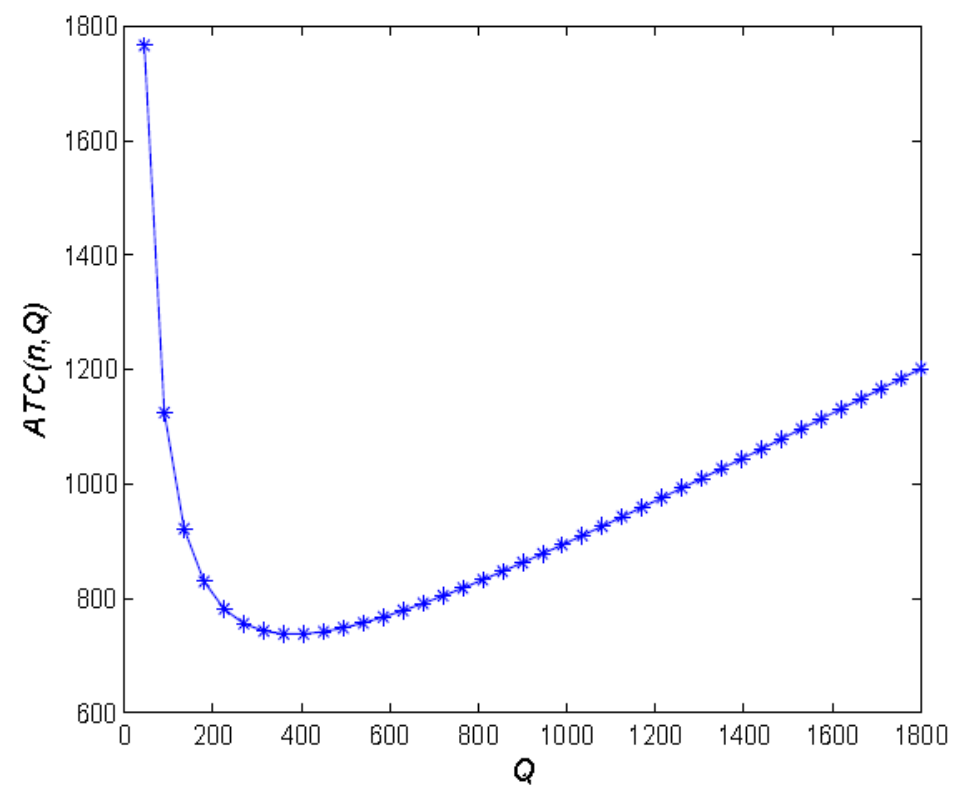

Fig. 2. Graphical representation of $A T C(n, Q)$ for $n=3$

The results obtained in the line search technique are shown in Table 1.

\section{Table 1}

Optimal results for different values of $n$ in Example 1

\begin{tabular}{ccc}
\hline$n$ & $Q^{*}$ & $\operatorname{ATC}\left(n, Q^{*}\right)$ \\
\hline 1 & 515.062 & 792.238 \\
2 & 379.459 & 737.058 \\
3 & $\mathbf{3 2 4 . 3 5 4}$ & $\mathbf{7 3 5 . 4 5 0}$ \\
4 & 295.765 & 751.958 \\
5 & 280.491 & 778.330 \\
6 & 273.889 & 811.796 \\
\hline
\end{tabular}

From the above table we see that the optimal number of shipments is 3 , the optimal order quantity of the buyer is 324.35 units and the corresponding average total cost is 735.45 units. Using Eq. (6), we then get the optimal cycle length of the buyer and that of the coordinated supply chain as $\tau^{*}=0.608$ unit and $T^{*}=1.824$ units, respectively.

Example 2. Uniform process shift distribution

Let $f(t)=\frac{1}{t_{m}}, \quad 0 \leq t \leq t_{m}$ and $f(t)=0$, otherwise. Then we have $E(N)=\int_{0}^{\frac{n Q}{p}} \alpha p\left\{\frac{n Q}{p}-t\right\} \frac{1}{t_{m}} d t=$ $\frac{\alpha n Q}{2}$. The optimal results of the proposed model are shown in Table 2. 
Table 2

Optimal results for different values of $n$ in Example 2

\begin{tabular}{ccc}
\hline$n$ & $Q^{*}$ & $A T C\left(n, Q^{*}\right)$ \\
\hline 1 & 515.773 & 712.574 \\
2 & 375.103 & 640.418 \\
3 & 315.211 & 620.262 \\
4 & $\mathbf{2 8 1 . 2 9 1}$ & $\mathbf{6 1 6 . 0 0 2}$ \\
5 & 259.657 & 618.917 \\
6 & 245.088 & 625.756 \\
7 & 235.136 & 635.009 \\
\hline
\end{tabular}

Example 3. Normal process shift distribution

Let $f(t)=\frac{1}{\sqrt{2 \pi \sigma^{2}}} \exp \left\{-\frac{(x-\mu)^{2}}{2 \sigma^{2}}\right\}, \quad 0 \leq t \leq t_{m} \quad$ and $f(t)=0$, otherwise. Then the expected number of defective items is given by $E(N)=\int_{0}^{\frac{n Q}{p}} \alpha p\left\{\frac{n Q}{p}-t\right\} \frac{1}{\sqrt{2 \pi \sigma^{2}}} \exp \left\{-\frac{(t-\mu)^{2}}{2 \sigma^{2}}\right\} d t=\alpha(n Q-p \mu)$

Taking $\mu=0.01$ and $\sigma=4$, we find the optimal decisions which are shown in Table 3 .

Table 3

Optimal results for different values of $n$ in Example 3

\begin{tabular}{ccc}
\hline$n$ & $Q^{*}$ & $A T C\left(n, Q^{*}\right)$ \\
\hline 1 & 515.061 & 792.237 \\
2 & 379.458 & 737.057 \\
3 & 324.354 & 735.452 \\
4 & 295.764 & 751.957 \\
5 & 280.491 & 778.330 \\
6 & 273.796 & 811.796 \\
\hline
\end{tabular}

We will now discuss some managerial implications in the case of numerical Example 1. Let us now consider the situation for $n=1$ i.e. lot for lot situation. If we consider the model from buyer's perspective, then the buyer's optimum order quantity is $Q_{b}^{*}=Q^{*}=275.55$ units and the average total cost is $\operatorname{ATC}_{b}^{*}=435.0414$ units. Substituting $Q^{*}=275.55$ in Eq. (10) we get $A T C_{v}^{*}=436.4919$ units. Then the average cost for the coordination becomes $A T C_{v}^{*}+A T C_{b}^{*}=871.5338$ units. This shows a cost reduction of 136.0838 units (871.5338-735.45) in the proposed supply chain model. Similarly, if we think of the model from vendor's perspective then we have the optimal order quantity $Q_{v}^{*}=Q^{*}=1010.43$ units and the average total cost $A T C_{v}^{*}=286.05$ units. Substitution of $Q^{*}=1010.43$ in Eq. (9) gives $A T C_{b}^{*}=598.69$ units. The average total cost in this non-coordinated policy is 884.74 units. This again shows that the coordination policy of the proposed supply chain model provides a cost reduction of 149.29 units for the lot-for-lot policy of the corresponding model. We now compare the results of the coordinated policy with the non-coordinated policy for different values of the parameters $b, \theta, \lambda$ and $\alpha$. For the non-coordinated policy, we follow the same procedure as above except taking $n=1$. It is easy to see from Table 4 that the coordinated cost is always lower than the non-coordinated cost for different values of the parameters. But the behavior of the cost reduction in the coordinated policy is not the same in all cases. 
Table 4

A comparison of the coordinated and non-coordinated policies for different values of modelparameters

\begin{tabular}{|c|c|c|c|c|c|c|c|}
\hline \multirow{2}{*}{ Parameter } & \multirow{2}{*}{ Value } & \multicolumn{3}{|c|}{ Coordinated policy } & \multicolumn{3}{|c|}{ Non-coordinated policy } \\
\hline & & $n^{*}$ & $Q^{*}$ & $\operatorname{ATC}\left(n^{*}, Q^{*}\right)$ & $n^{*}$ & $Q^{*}$ & $\operatorname{ATC}\left(n^{*}, Q^{*}\right)$ \\
\hline \multirow{5}{*}{$\theta$} & 0.010 & 2 & 406.72 & 715.09 & 1 & 302.25 & 832.01 \\
\hline & 0.015 & 2 & 392.37 & 726.26 & 2 & 259.08 & 753.01 \\
\hline & 0.020 & 3 & 324.35 & 735.45 & 1 & 275.55 & 871.53 \\
\hline & 0.025 & 3 & 314.82 & 744.54 & 2 & 238.00 & 778.89 \\
\hline & 0.030 & 3 & 306.09 & 753.37 & 2 & 229.22 & 791.19 \\
\hline \multirow{5}{*}{$\lambda$} & 50 & 3 & 321.81 & 733.34 & 2 & 246.63 & 777.54 \\
\hline & 75 & 3 & 323.50 & 734.75 & 2 & 247.45 & 765.24 \\
\hline & 100 & 3 & 324.35 & 735.45 & 2 & 247.87 & 767.18 \\
\hline & 125 & 3 & 324.86 & 735.87 & 2 & 248.12 & 767.01 \\
\hline & 150 & 3 & 325.19 & 736.15 & 2 & 248.28 & 767.10 \\
\hline \multirow{5}{*}{$b$} & 0.010 & 3 & 325.73 & 733.61 & 2 & 248.86 & 764.01 \\
\hline & 0.015 & 3 & 325.04 & 734.53 & 2 & 248.36 & 765.09 \\
\hline & 0.020 & 3 & 324.35 & 735.45 & 2 & 247.87 & 766.18 \\
\hline & 0.025 & 3 & 323.66 & 736.36 & 2 & 247.37 & 767.26 \\
\hline & 0.030 & 3 & 322.98 & 737.28 & 2 & 246.88 & 768.34 \\
\hline \multirow{5}{*}{$\alpha$} & 0.03 & 4 & 283.34 & 639.91 & 2 & 244.24 & 686.87 \\
\hline & 0.04 & 3 & 320.31 & 686.53 & 2 & 246.09 & 725.61 \\
\hline & 0.05 & 3 & 324.65 & 735.45 & 2 & 247.87 & 766.18 \\
\hline & 0.06 & 2 & 381.52 & 779.36 & 1 & 276.53 & 904.90 \\
\hline & 0.07 & 2 & 384.64 & 823.75 & 1 & 277.50 & 939.01 \\
\hline
\end{tabular}

When $\theta$ changes from 0.01 to 0.03 , the cost reduction in the coordinated policy increases an amount ranging from $5 \%$ to $15 \%$. For the parameters $\lambda$, and $b$, the cost reduction gradually decreases as the values of $\lambda$ and $b$ increase. On the on other hand, as $\alpha$ increases from 0.03 to 0.07 , the cost reduction first increases (attains a maximum of $17 \%$ when $\alpha=0.06$ ) and then gradually decreases.

\section{Conclusions}

This paper has considered a single-vendor single-buyer supply chain model where the consumption rate at the retailer depends on the on-hand stock and the production process at the manufacturer is not perfectly reliable. The process shift may occur during a production run. As a result, the machine produces some defective items which have significant impact on the coordinated policy, as shown in the numerical analysis. It is observed in the numerical study that the coordinated policy provides lower cost than the non-coordinated policy in all circumstances. Future study could consider analyzing the model for multi-item and/or multi-vendor/buyer situation.

\section{Acknowledgements}

The authors are thankful to the anonymous referee for his/her valuable comments and suggestions on improving the quality of the paper. The second author's work is partially supported by CSIR, New Delhi, Ref. No. 20-6/2009(i) EU-IV. 


\section{References}

Banerjee, A. (1986). A joint economic lot size model for purchaser and vendor. Decision Sciences, 17, 292-311.

Ben-Daya, M. \& Hariga, M. (2004). Integrated single vendor single buyer model with stochastic demand and variable lead time. International Journal of Production Economics, 92, 75-80.

Covert, P.R. \& Philip, G.C. (1973). An EOQ model for items with Weibull distribution deterioration. AIIE Transactions, 5, 323-326.

Ghare, P.N. \& Schrader, G.F. (1963). A model for exponentially decaying inventories. Journal of Industrial Engineering, 15, 238-243.

Goyal, S.K. (1976). An integrated inventory model for a single supplier-single customer problem. International Journal of Production Research, 15(1), 107-111.

Goyal, S.K. (1988). A joint economic lot size model for purchaser and vendor: a comment. Decision Sciences, 19, 236-241.

Goyal, S.K. (1995). A one-vendor multi-buyer integrated inventory model: a comment. European Journal of Operational Research, 81, 312-323.

Goyal, S.K. \& Giri, B.C. (2001). Recent trend in modeling of deteriorating inventory: an invited review. European Journal of Operational Research, 134, 1-16.

Hill, R.M. (1999). The optimal production and shipment policy for the single-vendor single buyer integrated production-inventory model. International Journal of Production Research, 37, 24632475.

Hogue, M.A. \& Goyal, S.K. (2000). An optimal policy for a single-vendor single buyer integrated production-inventory problem with capacity constraint of the transport equipment. International Journal of Production Economics, 65, 305-315.

Huang, C.K. (2004). An optimal policy for single-vendor single-buyer integrated productioninventory problem with process unreliability consideration. International Journal of Production Economics, 91, 91-98.

Kim, T., Hong, Y. \& Lee, J. (2006). Joint economic production allocation and ordering policies in a supply chain consisting of multiple plants and a single retailer. International Journal of Production Research, 43 (17), 3619-3632.

Li, J. \& Liu, L. (2006). Supply chain coordination with quantity discount policy. International Journal of Production Economics, 101, 89-98.

Lu, L. (1995). A one-vendor multi-buyer integrated inventory model. European Journal of Operational Research, 81, 312-323.

Moon, I. \& Lee, S. (2000). The effects of inflation and time value of money on an economic order quantity model with a random product life cycle. European Journal of Operational Research, 125, 588-601.

Nahmias, S. (1982). Perishable inventory theory: Operations Research, 30,680-708.

Qin, Y., Tang, H. \& Guo, C. (2007). Channel coordination and volume discounts with price sensitive demand. International Journal of Production Research, 105, 43-53.

Sajadieh, M.S., Akbari Jokar, M.R. \& Modarres, M. (2009) Developing a coordinated vendor-buyer model in two stage supply chains with stochastic lead times. Computers \& Operations Research, 36, 2484-2489.

Tadikamalla, P. R. (1978). An EOQ inventory model for items with gamma distributed deterioration. AIIE Transactions, 10, 100-103.

Wee, H. M. \& Chung, C.J. (2006). A two-echelon deteriorating production-inventory newsboy model with imperfect production process. Computational Science and its Applications, ICCSA Lecture Notes in Computer Science, 3982, 862-874.

Yang, M.F. (2010). Supply chain integrated inventory model with present value and dependent crashing cost is polynomial. Mathematical and Computer Modelling, 51, 802-809.

Zhou, Y.W., Min, J. \& Goyal, S.K. (2008). Supply chain coordination under an inventory-leveldependent demand rate. International Journal of Production Research, 113, 518-527. 